\title{
Operating in Configuration Space Significantly Improves Human Performance in Teleoperation *
}

\author{
I. Ivanisevic and V. Lumelsky \\ Robotics Lab, University of Wisconsin-Madison \\ Madison, Wisconsin 53706, USA \\ iigor@cs.wisc.edu
}

\begin{abstract}
This paper discusses the use of configuration space (C-space) as a means of visualization and control in teleoperation of robot arm manipulators. The motivation is to improve operator performance in tasks involving manipulator motion in a complex three-dimensional (3D) environment with obstacles. Unlike other motion planning tasks, operators are known to make expensive mistakes in arm control, due to deficiencies of human spatial reasoning. The advantage of $\mathrm{C}$-space is that in it the arm becomes a point, a case which humans are much better equipped to handle. To make such operation possible, a tool is proposed that reduces motion in 3D C-space to that in $2 \mathrm{D} \mathrm{C}$-space. It is then shown on results from testing 18 human subjects that translating the problem of a three-link $3 \mathrm{D}$ arm manipulator motion into C-space improves the operator performance remarkably, by a factor of 2 to 4 , compared to usual work space control.
\end{abstract}

\section{Introduction}

This paper is concerned with the development of a human-computer interface which would simplify motion planning tasks encountered in teleoperation of robot arm manipulators. It is a well known fact (this topic has been a subject of several experimental studies [1]) that human operators have a hard time controlling complex jointed devices such as robot arm manipulators. In the past, examples of applications of teleoperation have been reported (e.g. the NASA shuttle arm) where human operator error resulted in costly equipment damages and

*This work was supported by the Office of Naval Research Grant N000149710871. failure to complete the task.

This suggests a need for a machine intelligence that would assist the human in such tasks. In approaching this development task it is not clear, however, how the two intelligences - human and machine's - can be combined in a synergistic fashion. Studies [1] connect operator difficulties with peculiarities of human spatial reasoning: humans have difficulty handling simultaneous interaction with objects at multiple points of the device's body, or motion that involves mechanical joints (such as in arm manipulators), or dynamic tasks. With this in mind, our approach has been to use machine intelligence to reduce the task to one humans are known to be good at, i.e. a problem of moving a point in a maze.

Our visual human-computer interface transforms the task of controlling a complicated jointed arm manipulator (the work space (W-space) control) to one of control of a point in the corresponding configuration space (C-space). Even in a simpler 2D case control in C-space was shown [2] to improve operator performance rather remarkably, and even outperform the best known algorithms running on modern workstations. Results in $2 \mathrm{D}$, however, are not easily translated to $3 \mathrm{D}$ tasks which typical real-world applications would require [3]; that operation is the subject of this paper. As shown below, a direct translation of the 2D approach does not work and an additional mapping is needed to overcome this problem.

Below, the properties of the arm model and its C-space are discussed in Section 2, the proposed interface appears in Section 3, and the experimental setup, results and discussion are given in Section 4 . 


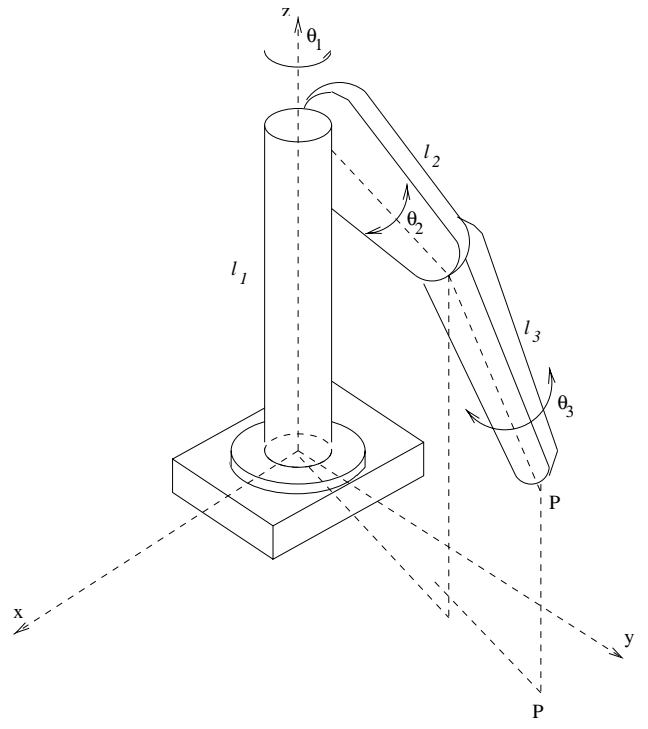

Figure 1: The 3D RRR arm manipulator.

\section{The arm}

Arm Geometry and Positioning. We consider a three-link three-dimensional arm manipulator with three revolute joints (RRR arm). The arm operates in an environment with stationary obstacles, Figure 1. The arm's first link, $l_{1}$, rotates about a vertical axis, producing joint values $\theta_{1}$. Each of the other two links, $l_{2}$ and $l_{3}$, rotates in a vertical plane; their positions are defined by the values $\theta_{2}$ and $\theta_{3}$, respectively. Joint values are limited by mechanical stops, $\left|\theta_{3}\right|<2 \pi$. For computational efficiency links are modeled as generalized cylinders. Note that link $l_{2}$ is attached to the side of base $l_{1}$, and link $l_{3}$ is attached to the side of link $l_{2}$ to allow $360^{\circ}$ motion. The virtual arm used in the experiments (Section 4) is shown in Figure 2.

Configuration Space (C-space). The arm's position is thus uniquely defined by the triple $\left(\theta_{1}, \theta_{2}, \theta_{3}\right)$. All possible values of the latter form the configuration space (C-space) of the arm manipulator (for application of C-space to robot motion planning see e.g. [4],[5]).

Since in our scheme the operator will control the robot in C-space, the latter has to be computed first. A position in $\mathrm{W}$-space in which the arm touches some obstacle maps in C-space uniquely into a point on the surface of the corresponding obstacle image. The set of all positions in $\mathrm{W}$-space in which the arm touches one or more obstacles will form the surface(s) of C-space obstacles. The

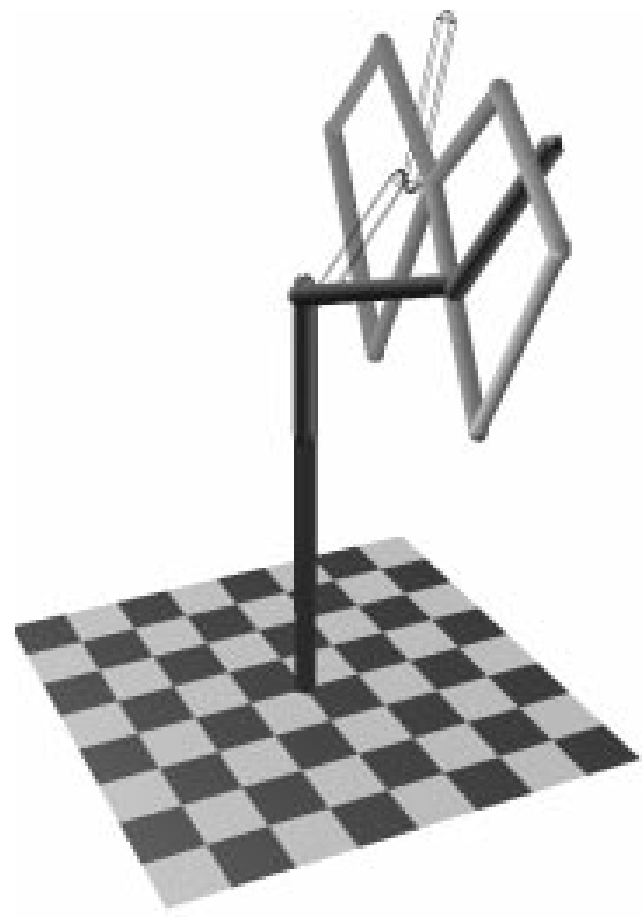

Figure 2: A sample task in W-space (Task 1 in Section 4).

said mapping is not linear and so C-space obstacles look very different from $\mathrm{W}$-space obstacles.

\section{Visual Feedback and Control}

We can now compare two types of visual feedback that that one can realize - in W-space or in $\mathrm{C}$-space. Their use in tests with human subjects is discussed in Section 4.

W-space Feedback and Control: In this scheme, the user is presented with an image of the three-dimensional W-space, complete with the arm and any physical obstacles in the scene. See an example in Figure 2: the darker object is the arm, the lighter object - a relatively complex obstacle. The starting position of the arm is shown in solid, and its target position is shown as a skeleton arm. The user can manipulate the W-space image interactively, including zoom in and zoom out, and changing the position of the "camera" (i.e. the viewer's position relative to the scene). (Note that such flexibility of control is feasible in virtual scenes but is not likely to be reproducible in a physical system because it would require either a 


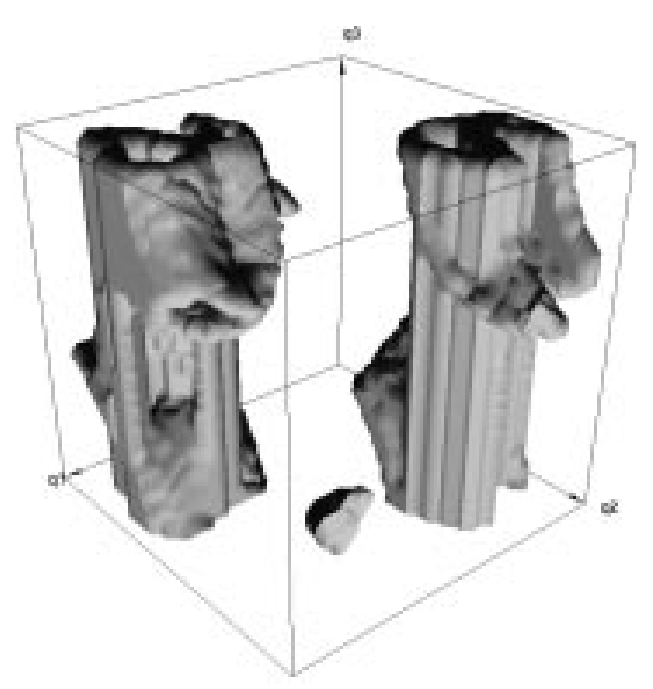

Figure 3: Three-dimensional C-space of the sample task in Figure 2.

very large number of cameras or a possibility of an arbitrary positioning for the viewing camera(s)).

In an attempt to provide a good interface, an input device has been built, which presents an arm resembling the one on the screen. The operator can use this master arm to guide the virtual slave arm on the screen while receiving continuous visual feedback from the "camera". Given the difficulty the operators have with arm control in the vicinity of obstacles, additional visual feedback means has been provided, which in a way simulates a haptic interface. A small bright sphere appears on the point(s) of the virtual arm body that comes into contact with an obstacle. Also, if after the contact the operator continues moving the arm "into the obstacle", a skeleton arm figure will follow the actual motion while the solid arm will stay at the point of contact.

\section{C-space Feedback and Control.}

As explained above, transforming the problem of moving a complex jointed arm (in W-space) to that of moving a simple point (in C-space) makes the task much easier for the humans. But, another problem, which did not appear in the two-dimensional case [2], appears in the 3D case. Namely, for nonpoint objects various means (e.g. perspective projections, shading etc.) can be used to help estimate the object's location. These are not useful for a point: when moving a point on the screen, it is very hard to see where it is located in a $3 \mathrm{D}$ labyrinth. A no less serious problem is that the operator cannot see most of the interior of C-space obstacles, which tend to be cave-like structures, with multiple entrances, branches and dead-ends inside (see Figure 3). Attempts to "go inside" those caves would likely lead to an exhaustive search of the insides of the C-space obstacles.

This suggests another mapping, which would transform the 3D C-space to some two-dimensional space, while retaining information about $\mathrm{C}$-space obstacles. The mapping introduced here is based on the concept of $C$-space slicing, which is not unlike the slicing used in earlier work on motion planning [4]. Assume each side of the C-space cube is $2 \pi$ long; if a joint - say, $\theta_{1}$ - has limits, $\left(\theta_{1 \text { min }}, \theta_{1 \text { max }}\right)$, then the parallelepiped in $\mathrm{C}$-space between $\theta_{1}=\theta_{1 \text { min }}, \theta_{1}=\theta_{1 \text { max }}$ is a range obstacle which, from the motion standpoint, is as any other obstacle. [C-obstacles shown in Figure 3 represent only physical obstacles; range obstacles are omitted].

We slice C-space bottom up, along the $\theta_{3}$ axis; the result is a number of slice squares each representing a $\theta_{3}$ slice. Each side of each square represents the $2 \pi$ range of $\theta_{1}, \theta_{2}$, respectively. A slice may include regular $\mathrm{C}$-space obstacles and range obstacles if any. If the range of $\theta_{3}$ is $\theta_{3 \min }$ to $\theta_{3 \max }$ and the slice thickness is $\Delta \theta_{3}$, then the number of slices is $m=\left(\theta_{3 \min }-\theta_{3 \max }\right) / \Delta \theta_{3}$. Smaller $\Delta \theta_{3}$, will results in better resolution and bigger $m$. The maximum $m$ is determined by the screen size and operator's convenience. Figure 4 shows the slice mapping of C-space of Figure 2.

Rectangular shaded stripes in the squares (including fully shaded squares in the middle of the figure) correspond to the range obstacles, more "irregular" shapes - to normal C-obstacles. The 100 squares shown correspond to the resolution of 3.6 degrees along the $\theta_{3}$ axis. In the figure, the bottom left corner corresponds to $\theta_{3}=0$ and the upper right corner to $\theta_{3}=2 \pi-\Delta \theta_{3}-$ which means these slices are actually neighbors.

In each row slices go from left to right, so that the leftmost slice in row $n$ follows the rightmost slice in the row $n-1$ and vice versa. $\mathrm{S}$ and $\mathrm{T}$ mark the start and target positions corresponding to those in Figure 2. When working in C-space, the operator moves the point representing the arm's current position within a slice or between neighboring slices. One can also try to jump over many slices - the computer will allow such moves if the (C-space) straight line between the current and intended positions does not cross any obstacles. To 
increase visibility of details (like narrow openings etc.), one can zoom-in at a given slice by clicking the mouse (this makes the slice occupy the whole screen) and zoom-out back when needed.

\section{Experimental Results and Discus- sion}

In the experiment, 18 human subjects have been tested in the same motion planning tasks with a three-link RRR arm manipulator. The objective was to compare the subjects' performance in W-space and C-space using the two interfaces described in Section 3. Each subject was tested on two tasks - an easier task (Task 1, Figure 2) and a task (Task 2) that was meant to be more of a challenge to human spatial reasoning. The experiment consisted of two parts completed on separate days, with Part 1 followed by Part 2.

Part 1: the task is to plan the arm motion from the starting to the target position in C-space, using the C-space interface. Each subject completed Task 1 shown in Figure 4, and then a more complex Task 2 (not shown). Prior to the test each subject went through a training period of about 15 min during which they would familiarize themselves with the interface and solve a simple training example; for additional comparison, three subjects received more extensive training.Performance was measured by the length of the path produced, determined as the sum of changes of the three joint values $\left(\theta_{1}, \theta_{2}, \theta_{3}\right)$ and the time taken to complete the task (in seconds).

Part 2: Here the subjects were asked to solve the problem using the $\mathrm{W}$-space interface. Once again, the subjects received 15 min of training during which they would solve a simple training example (again, the same three subjects received more extensive training). Each subject would then complete Task 1 shown in Figure 2, followed by the W-space version of Task 2 used in C-space. As before, subjects' performance was measured by the path length and time to completion using the same metric.

Discussion: Tables 1 and 2 summarize the subjects' performance in W-space vs. C-space, in Task 1 and Task 2, respectively, in terms of path length produced (e.g. W-path is the path length produced using the $\mathrm{W}$-space interface) and time
Table 1: W-space vs. C-space, Task 1 statistics

\begin{tabular}{|c|c|c|c|c|}
\hline & W-path & C-path & W-time & C-time \\
\hline \hline Mean & 7.020 & 2.759 & 374.278 & 229.167 \\
\hline Min. & 2.237 & 1.193 & 151 & 39 \\
\hline Max. & 17.803 & 5.590 & 847 & 566 \\
\hline Std. Dev. & 4.643 & 1.256 & 246.270 & 133.843 \\
\hline
\end{tabular}

Table 2: W-space vs. C-space, Task 2 statistics

\begin{tabular}{|c|c|c|c|c|}
\hline & W-path & C-path & W-time & C-time \\
\hline \hline Mean & 8.065 & 2.241 & 569.667 & 158.667 \\
\hline Min. & 3.702 & 1.480 & 170 & 71 \\
\hline Max. & 20.434 & 2.995 & 1406 & 316 \\
\hline Std. Dev. & 3.990 & 0.418 & 280.968 & 64.572 \\
\hline
\end{tabular}

taken to complete the task. Figure 5 shows a typical example of performance in C-space in Task 1; Figure 6 shows the same for W-space. The dark lines show the path of the arm endpoint; also shown is the projection of the path on the floor ( $x y$ plane).

Note a remarkable improvement in subjects' performance in C-space compared to W-space. As seen in Tables 1 and 2, the mean path length produced in C-space is roughly 2.5 times better (shorter) than in W-space for Task 1, and more than 3.5 times better for Task 2. Furthermore, both the best and the worst performances are better in C-space than in W-space (see the min and max columns of Tables 1-2). In fact, only in one instance, in Task 1, one subject performed (marginally) better in W-space than in C-space (producing a path of length 5.21 as compared to 5.59 , respectively). In Task 2 the same subject did significantly better in C-space as compared to Wspace.

One parameter that was not measured, but could be of great interest in applications, was the number of times the arm hit an obstacle during the task execution. Based on our observations, this number was much higher when working in $\mathrm{W}$-space than in C-space. Overall, the subjects found it much easier to work, and they performed admirably, in C-space compared to the $\mathrm{W}$-space. This difference was even more evident with the three subjects who received longer training with both interfaces.

In C-space Task 1, the three well-trained subjects' mean path length was 1.52 and the mean time $86.3 \mathrm{sec}$. For the same task these numbers in $\mathrm{W}$-space were 4.36 and $240.7 \mathrm{sec}$, respectively. 


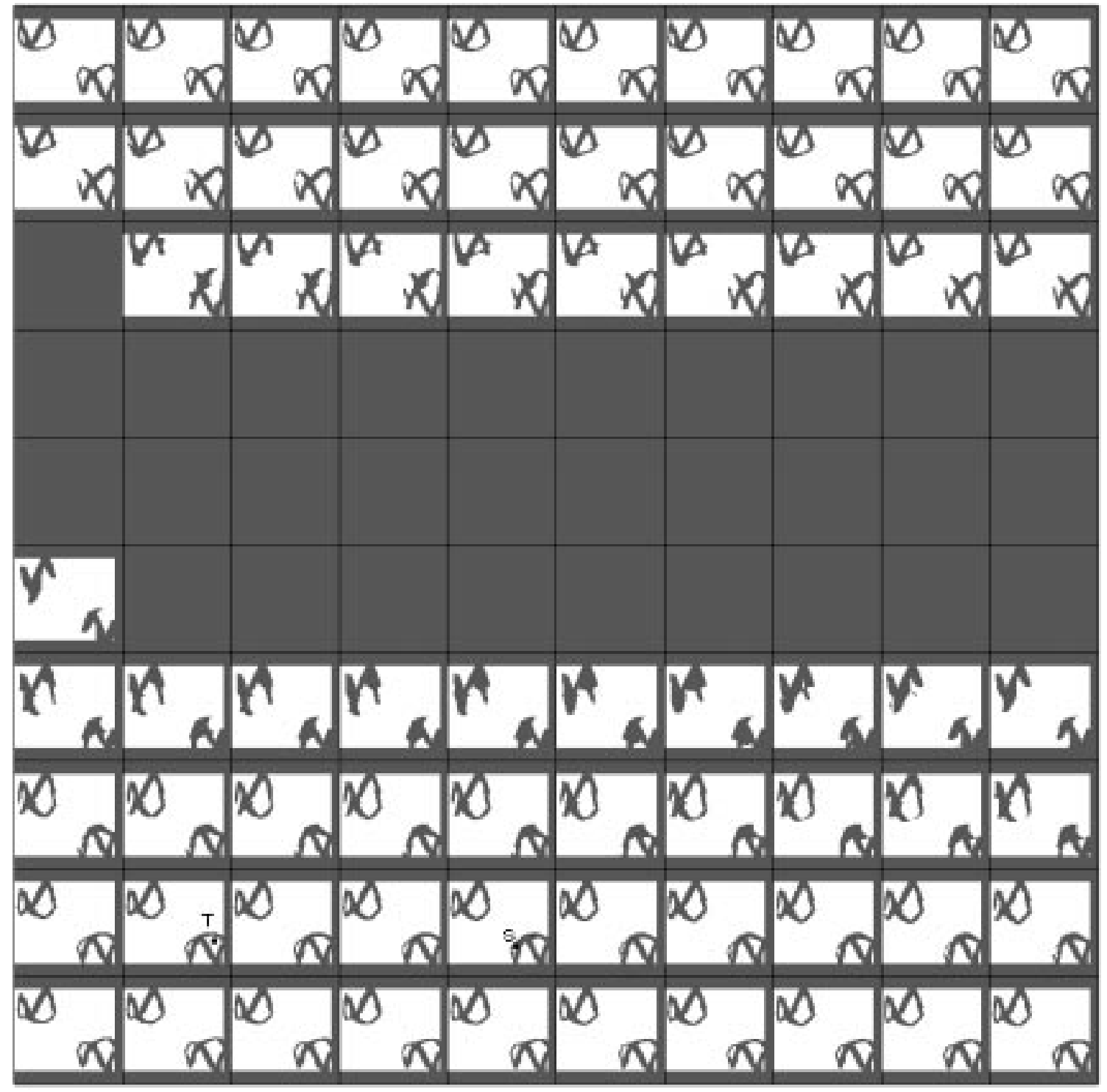

Figure 4: Slicing of the 3D C-space in Figure 3. This also corresponds to the W-space in Figure 2.

When considering those numbers, one should take into the account that the optimal path for Task 1 has a length of about 1.10. That is, in C-space the three well-trained subjects produced a nearly optimal path. On the other hand, when working in W-space, their performance was significantly worse than that of an average little-trained subject in C-space (with 15 min training), albeit significantly better than that of little-trained subjects in $\mathrm{W}$-space.

In C-space Task 2, the well-trained subjects' mean path length was 1.63 and the mean time was $92.7 \mathrm{sec}$. For the same task these numbers in $\mathrm{W}$-space were 7.77 and $591.7 \mathrm{sec}$, respectively.
Note that the path length 7.77 is only marginally better than the mean of all subjects - training did not help the well-trained subjects in this case. In this more complicated task, the optimal path length is about 1.35. On the other hand, since the performance of the well-trained subjects in C-space was consistently better than of the little-trained subjects, one can conclude that training does in fact help in that case. Given the overhead of effort necessary to understand the more complex rules of operation in C-space and getting used to the unfamiliar shapes of C-space obstacles, it is reasonable to suggest that training will, in general, significantly improve operator performance in $\mathrm{C}$-space. 


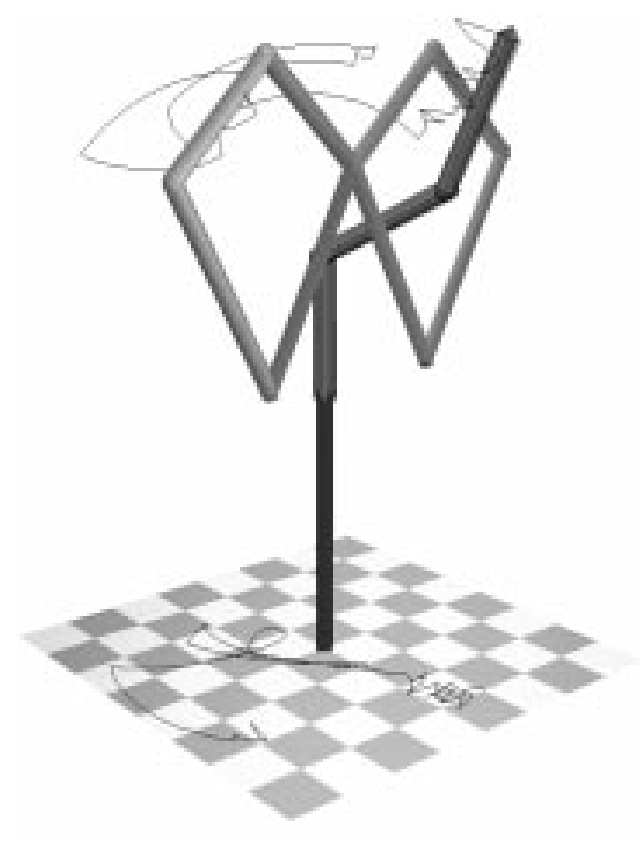

Figure 5: A typical W-space path produced using the C-space interface in Task 1.

\section{Conclusion}

This paper proposes an approach to humanguided teleoperation of a robot arm manipulator based on operation in the task's configuration space rather than in the commonly used $\mathrm{W}$-space. To improve the human interface, a tool is proposed for reducing the motion in $3 \mathrm{D} \mathrm{C}$-space to that in $2 \mathrm{D}$ C-space. With it, a two-dimensional, sliced version of the three dimensional $\mathrm{C}$-space is offered to the operator. Instead of directly confronting the problem of collision analysis, which is known to be extremely challenging for the human spatial reasoning, the operator faces the task in C-space where one can concentrate on global navigation while easily avoiding collisions. Thus reduced task becomes a variant of the maze-searching problem. Our experiments with human subjects show that in spite of the strange appearance of this new interface, subjects quickly learn it and perform in it remarkably better than in the familiar commonly used work space.

The authors express their gratitude to Jon Lawrence for the help in designing and building the master arm used in this work.

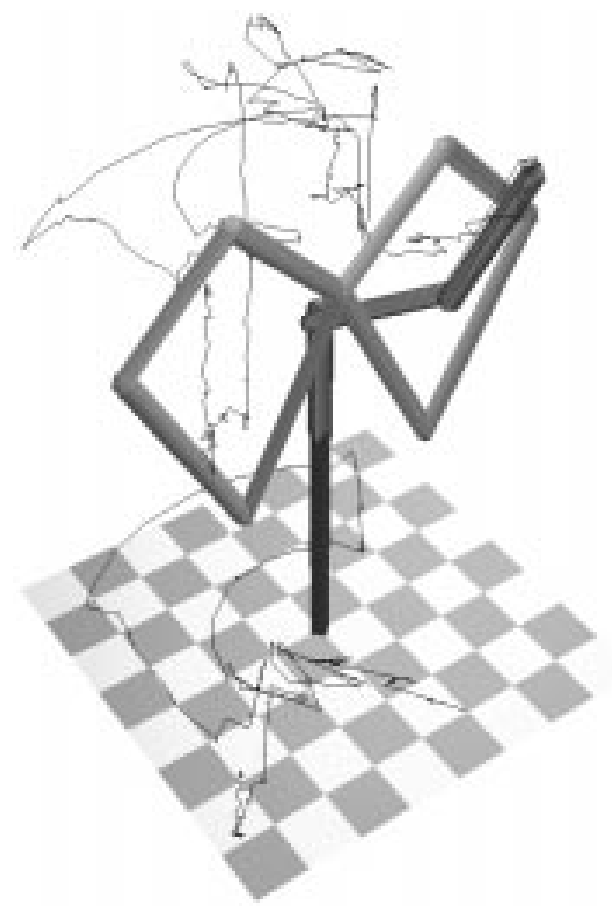

Figure 6: The typical W-space path produced using the W-space interface in Task 1.

\section{References}

[1] F. Liu, Multivariate Analysis of Human Performance in Motion Planning. M.S. Thesis, University of Wisconsin-Madison, Mechanical Engineering, May 1997.

[2] I. Ivanisevic, V. Lumelsky, A Human-Machine Interface for Teleoperation of Arm Manipulators in a Complex Environment, Proceedings of the 1998 IEEE International Conference on Intelligent Robots and Systems, October 1998.

[3] Q. Lin, C. Kuo, Virtual Teleoperation of Underwater Robots, Proceedings of the 1997 IEEE International Conference on Robotics and Automation, April 1997.

[4] T. Lozano-Perez, Spatial planning: a configuration space approach, IEEE Transactions on Computers, Vol. 32, No. 3, February 1983.

[5] J. Schwartz, M. Sharir, On the Piano Movers problem. Part II. General Techniques for Computing Topological Properties of Real Algebraic Manifolds, Advances in Applied Mathematics, Vol. 4, pp. 298-351, 1983. 